\title{
An evidence-informed strategy to prevent osteoporosis in Australia
}

\author{
An outline of the Building healthy bones throughout life white paper
}

Peter R Ebeling MB BS, MD, FRACP Endocrinologist

Robin M Daly BAppSci(Hons), PhD, FASMF,

Chair in Exercise and

Ageing

Deborah A Ker PhD, MSc, GradDipDiet, Associate Professor of Nutrition and Dietetics

Michael G Kimlin $\mathrm{PhD}$ Professor ${ }^{4}$

1 NorthWest Academic Centre, University of Melbourne, and Western Health, Melbourne, VIC

2 Centre for Physical Activity and Nutrition Research, Deakin University, Melbourne, VIC

3 Curtin Health Innovation Research Institute and School of Public Health Curtin University, Perth, WA

4 Faculty of Health Queensland University of Technology, Brisbane, QLD.

peterre@

unimelb.edu.au

doi: 10.5694/mjal2.11363

This is an adapted, abridged version of the fully referenced and peer-reviewed white

paper published on www.mja.com.au/open

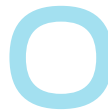

steoporosis currently affects 1.2 million Australians, most of whom do not know they have the disease. Without intervention, this is expected to increase to 3 million by 2021 as a result of population ageing. There are also now 6.3 million Australians with osteopenia. General practitioners and their patients often overlook bone health and, as a result, osteoporosis is often not diagnosed until fragility fractures occur. There is also a lack of an accepted strategy for osteoporosis prevention in Australia. Currently, treatment is based on bone mineral density (BMD) measurement and/or a prior fracture. However, more than $50 \%$ of women and $70 \%$ of men who sustain fragility fractures do not have BMD in the osteoporosis range (T score $<-2.5)$. This represents a "prevention paradox", which is the basis for developing the population-based prevention strategy outlined here.

Non-modifiable factors that increase the risk of osteoporosis include being female, menopause, age, certain medical conditions, and a genetic predisposition. Modifiable risk factors include lack of weight-bearing exercise, poor calcium intake, vitamin D deficiency, low or high body weight, cigarette smoking, excessive alcohol use, and long-term use of corticosteroids.

In 2011, Osteoporosis Australia held a Summit to develop clear, succinct, evidence-informed recommendations about calcium, vitamin D and exercise requirements for building healthy bones. The Summit and its recommendations are detailed in a fully referenced white paper, Building healthy bones throughout life, online at http://www.mja.com.au/open, and a précis of the white paper is provided as a Supplement to this issue of the Journal.

Building healthy bones throughout life provides the evidence for three affordable and important ways of reducing the enormous personal and economic costs of osteoporosis. These interventions are ensuring people have (1) an adequate calcium intake, (2) an adequate serum level of vitamin D, and (3) appropriate physical activity throughout their lives. As the relative importance of these interventions and the need for supplementation changes throughout life, separate recommendations are given for children, adults, and older adults, as well as individuals with osteoporosis. The core message is that adequate dietary calcium intake and optimal vitamin D level, together with regular weight-bearing exercise and moderate sunlight exposure, are important at all stages of life in healthy individuals.

\section{Calcium}

The median dietary intake of calcium in the last Australian National Nutrition Survey was $827 \mathrm{mg}$ per day for men and
$663 \mathrm{mg}$ per day for women; thus men's calcium intake was close to the estimated average requirement (EAR) of $840 \mathrm{mg}$ per day, but women fell well short. Most systematic reviews favour calcium plus vitamin D supplementation to reduce fracture risk, with an overall benefit of $10 \%-20 \%$ compared with placebo, while even greater reductions in fracture risk $(\geqslant 30 \%)$ have been observed in older people. The combination of vitamin $\mathrm{D}$ with calcium has also been shown to reduce mortality by $7 \%$.

Children: The aim with calcium intake in children and adolescents is to optimise peak bone mass ("bone in the bank"). During infancy, calcium provided by breast milk is assumed to be adequate and is the basis for most recommended intakes in the first few months of life. However, findings from the 2007 Australian National Children's Nutrition and Physical Activity Survey indicated that girls aged 12-16 years and boys aged 14 16 years were most at risk of not meeting their daily calcium dietary requirements $(82 \%-89 \%$ of girls and $44 \%$ of boys did not meet the EAR). It was suggested that this is likely due to a substantial decline in milk intake in childhood and its replacement with carbonated beverages. Systematic reviews of randomised controlled trials (RCTs) of calcium supplements in children suggest that increasing calcium intake from an average $700 \mathrm{mg}$ per day to $1200 \mathrm{mg}$ per day has limited benefits for bone mass. The evidence therefore does not support the use of calcium supplements in healthy children, with the possible exception of those with very low intakes ( $<700 \mathrm{mg}$ per day).

Healthy adults: There are only limited studies into the role of calcium intake in maintaining bone mass or fracture prevention in young and middle-aged adults. An adequate calcium intake achieved through diet (3-4 servings of calcium-containing foods each day) continues to be the best choice for those who can include an adequate intake of dairy products. To achieve the recommended intakes of $1000-1300 \mathrm{mg}$ per day, most adults would routinely need to also include at least one serving of a calcium-fortified food. There is no evidence that individuals consuming calcium at significantly higher levels receive any additional skeletal benefit.

Older adults: As there is evidence that the calcium needs in this group are greater and nutrition can also be more precarious, supplementation is a key strategy to ensure adequate calcium levels. Systematic reviews of RCTs aimed at preventing fractures show calcium can help prevent osteoporosis and fragility fractures. However, there is significant variation in the results because of differences in dosage, baseline nutrient status and the co-administration of vitamin D, as well as poor adherence to the supplements. The frail elderly have the highest rates of fracture, and those in residential care typically 
have vitamin D deficiency and inadequate calcium intake. The evidence is mixed but, on balance, shows that calcium supplementation prevents fractures in the frail elderly, particularly women in residential care.

Recent reanalyses of adverse events from some trials have suggested the use of calcium supplements may be associated with an increased risk of cardiovascular events. These claims of increased myocardial infarction (MI) risk have generated considerable scientific debate and a re-evaluation of the riskbenefit analysis of calcium supplementation. The conclusion of Building healthy bones throughout life is that, as none of the trials reanalysed were primarily designed to investigate cardiovascular outcomes, on balance, current evidence does demonstrate a small increase in risk of MI with calcium supplements. Using Women's Health Initiative data, based on the worst-case scenario, treatment of 1000 people with calcium or calcium and vitamin D for 5 years would cause an additional six MIs or strokes and prevent three fractures. However, mortality is not increased and, in fact, the combination of vitamin $\mathrm{D}$ with calcium supplements has been found to reduce mortality in the elderly by $7 \%$. If calcium and vitamin D are taken more than $80 \%$ of the time, the prevalence of fractures has been found to decrease by $24 \%$ in older men and women. Dietary calcium is the preferred source, and calcium supplements should be limited to 500-600 mg per day.

\section{Vitamin D}

Vitamin D adequacy is important for bone and muscle function. Vitamin D deficiency (serum 25-hydroxyvitamin D [25(OH)D] level $<50 \mathrm{nmol} / \mathrm{L}$ in late winter/early spring) is common in Australia. The findings from a national population-based sample of 11218 Australian adults aged 25-95 years showed that $31 \%$ of the population had a serum $25(\mathrm{OH}) \mathrm{D}$ level $<50 \mathrm{nmol} / \mathrm{L}$, and that the prevalence of deficiency increased with age and was greater in women than men, in those of non-European ancestry, and in those living in the southern states, particularly during winter.

Older people, particularly those living in residential aged care facilities, are at high risk of vitamin D deficiency. Others at greatly increased risk of vitamin D deficiency include: people with dark skin; those who wear modest dress; people at high risk of skin cancer because of past history or immunosuppression; people with intestinal malabsorption of key nutrients; and people less likely to spend time in the sun for health or work-related reasons.

Sunscreen use in the general population is not associated with low vitamin D levels, despite sunscreen's theoretical capacity to block most ultraviolet B radiation. Inadequate application combined with higher sun exposure in people who use sunscreen probably explains the discrepancy.

Pregnancy and childhood: Recent Australian research has confirmed the high prevalence of low vitamin D levels in pregnant women, and the potential adverse effects on fetal bone health and other pregnancy outcomes. As such, it is reasonable to check vitamin $\mathrm{D}$ status in all pregnant women and supplement to achieve maternal serum 25(OH)D levels $>50 \mathrm{nmol} / \mathrm{L}$, although the question of whether empirical supplementation without testing would be cost-effective remains unanswered.

Vitamin D is important for bone health and muscle function throughout childhood and adolescence. Adequate vitamin D status is required to prevent rickets and to promote normal bone growth and mineralisation as peak bone mass is acquired. Based on available evidence, the recommended level of $25(\mathrm{OH}) \mathrm{D}$ for infants, children and adolescents for optimal bone health remains at $>50 \mathrm{nmol} / \mathrm{L}$ year round.

Adults and individuals with osteoporosis: Both the recent position statement on vitamin $\mathrm{D}$ and health in adults from the Australian and New Zealand Bone and Mineral Society, the Endocrine Society of Australia and Osteoporosis Australia and the recent United States Institute of Medicine (IOM) report on dietary reference intakes for calcium and vitamin $\mathrm{D}$ concluded that inadequate vitamin $\mathrm{D}$ status is defined as a $25(\mathrm{OH}) \mathrm{D}$ level $<50 \mathrm{nmol} / \mathrm{L}$ at the end of winter. Levels of $25(\mathrm{OH}) \mathrm{D}$ may need to be $10-20 \mathrm{nmol} / \mathrm{L}$ higher at the end of summer to allow for seasonal variation. Systematic reviews of trials of vitamin D supplementation to prevent falls and fractures have found serum $25(\mathrm{OH}) \mathrm{D}$ thresholds should be $60 \mathrm{nmol} / \mathrm{L}$ and $75 \mathrm{nmol} /$ L, respectively. A recent Endocrine Society clinical practice guideline recommends that adults aged $50-70$ years and those over 70 years require at least $600 \mathrm{IU}$ and $800 \mathrm{IU}(15 \mu \mathrm{g}$ and $20 \mu \mathrm{g}$ ) of vitamin $\mathrm{D}_{3}$ per day, respectively, to maximise bone health and muscle function. However, to raise the serum $25(\mathrm{OH}) \mathrm{D}$ level $>75 \mathrm{nmol} / \mathrm{L}$, as both the Endocrine Society and the International Osteoporosis Foundation recommend, may require at least $1500-2000 \mathrm{IU}(37.5-50 \mu \mathrm{g})$ per day of supplemental vitamin $\mathrm{D}$, while for severe deficiency, doses $\leqslant 10000 \mathrm{IU}(250 \mu \mathrm{g})$ per day have proven to be safe.

\section{Exercise}

Regular physical activity and exercise is recognised as one of the most effective lifestyle strategies to maximise peak bone mass during growth and prevent bone loss during ageing. However, the osteogenic benefits of exercise depend on stage of life and the relative risk of fracture. Childhood and adolescence may represent the optimal time in which exercise can improve bone strength and protect against osteoporosis and fragility fractures in old age, particularly when these early benefits can be maintained by adopting appropriate measures in later life. A 10\% higher peak bone mass can delay the development of osteoporosis by 13 years and reduce the risk of fracture by $50 \%$.

Exercise programs that combine high-impact activity with high-intensity resistance training appear most effective in augmenting BMD in premenopausal women. High-impactalone protocols (eg, jumping) are effective only on hip BMD in this group. In frail and very elderly adults, resistance training and balance exercises in combination reduce falls and risk factors for frailty, including sarcopenia, poor balance, gait instability, depression, fear of falling and cognitive impairment. Multimodal exercise, including weight-bearing, high-impact and high-intensity resistance exercise, may significantly reduce overall fracture risk. By contrast, singlemodality exercise of any type does not appear to reduce fracture risk, with the possible exception of spinal-extensor muscle resistance training, which reduces thoracic vertebral fracture incidence.

Acknowledgements: Published with the Building healthy bones for life white paper on www.mja.com.au/open.

Competing interests: Peter Ebeling has received prior research support from Sanofi and currently receives research funding from MSD, Amgen, Novartis and Eli Lilly. He has previously received honoraria from Merck and a travel grant from DiaSorin to present at a meeting. Robin Daly has received a prior honorarium from Merck.

Provenance: Commissioned. 